\title{
Analysis of the IBEX-Lo interstellar hydrogen fluxes collected in 2009-2018 as a tool for sensing of the solar radiation pressure and the hydrogen ionization rate
}

\author{
O. A. Katushkina ${ }^{\circledR},{ }^{1 \star}$ A. Galli, ${ }^{2}$ V. V. Izmodenov ${ }^{\circledR 1,3,4}$ and D. B. Alexashov ${ }^{1,5}$ \\ ${ }^{1}$ Space Research Institute of Russian Academy of Sciences, Profsoyuznaya Str 84/32, Moscow 117335, Russia \\ ${ }^{2}$ Physics Institute, University of Bern, Bern CH-3012, Switzerland \\ ${ }^{3}$ Moscow Center for Fundamental and Applied Mathematics, Lomonosov Moscow State University, GSP-1, Leninskie Gory, Moscow 119991, Russia \\ ${ }^{4}$ National Research University Higher School of Economics, Physical Department, Myasnitskaya Str. 20, Moscow 101000, Russia \\ ${ }^{5}$ Institute for Problems in Mechanics, prosp. Vernadskogo 101, block 1, Moscow 119526, Russia
}

Accepted 2020 December 2. Received 2020 December 2; in original form 2020 October 23

\begin{abstract}
The Interstellar Boundary Explorer (IBEX) has been measuring interstellar hydrogen fluxes at 1 au since 2009. In this paper, we analysed all available data obtained with the IBEX-Lo instrument at energies $11-41 \mathrm{eV}$ using our numerical kinetic model of the interstellar hydrogen distribution in the heliosphere. We performed a fitting of the data to find independently the model parameters: the ratio of the solar radiation pressure to the solar gravitation $\left(\mu_{0}\right)$, ionization rate of hydrogen atoms at 1 au $\left(\beta_{0}\right)$, parameters of the secondary interstellar atoms at 70 au from the Sun, which provide the best agreement with the data by minimization of metric $\chi^{2}$. We also analysed temporal variations of the ratio of the fluxes measured in a fixed direction at energy bin 1 and energy bin 2. It is found that in 2009-2011 and 2017-2016 the ratio provided by the model is smaller than in the IBEXLo data, while in 2012-2015, oppositely, the model ratio is larger compared to the data. This might be caused by the incorrect separation of the measured fluxes between energy channels in the data, or by some additional physical factors that are omitted in the model. Understanding this issue may be important for the preparation of future Interstellar Mapping and Acceleration Probe mission. At this stage, we relied on the sum of the fluxes measured in energy bins 1 and 2 for comparison to model predictions.
\end{abstract}

Key words: Sun: heliosphere-solar wind-ISM: atoms.

\section{INTRODUCTION}

The Interstellar Boundary Explorer (IBEX) mission was launched to the Earth's orbit in 2008 and has been measuring fluxes of the interstellar and the heliospheric energetic neutrals during more than $10 \mathrm{yr}$ since 2009 up to now. The objective of the mission is remote imaging of the heliospheric boundary by 3D mapping of the energetic neutral atoms (ENAs) created around the heliopause that is the boundary between the solar wind flow and the surrounding interstellar matter (McComas et al., 2009, Science). Besides ENAs IBEX provides a unique opportunity for direct sampling of the interstellar low energetic neutrals coming from the pristine interstellar matter. The IBEX payload consists of two instruments - IBEX-Lo (energies from $10 \mathrm{eV}$ to $2 \mathrm{keV}$, Fuselier et al., 2009) and IBEX-Hi (energies from $300 \mathrm{eV}$ to $6 \mathrm{keV}$, Funsten et al., 2009). Both instruments have several energy channels. ENAs created in the region of interaction of the solar wind and the interstellar medium have energies in the range of $\sim 0.1-5 \mathrm{keV}$, and they can be detected at high-energy channels of IBEX-Lo and all energy channels of IBEX-Hi. The interstellar neutral (ISN) hydrogen atoms have energies $\sim 10-40 \mathrm{eV}$, and they can be detected only by low channels of IBEX-Lo.

The ISNs of $\mathrm{H}, \mathrm{He}, \mathrm{O}, \mathrm{Ne}$, and $\mathrm{D}$ originate from the local interstellar medium (LISM) surrounding our Solar system. The

^E-mail: okat@iki.rssi.ru interstellar medium is a partially ionized hydrogen plasma with a minor component of other species. The Sun is moving through the LISM with a relative velocity of $\sim 26 \mathrm{~km} \mathrm{~s}^{-1}$ (Möbius et al., 2004; McComas et al., 2015). The ISNs have a large mean free path and therefore they penetrate through the region of interaction between the solar wind with the charged component of the LISM. On the way from the undisturbed interstellar medium to the heliosphere some atoms charge exchange with the interstellar ions. By exchanging an electron, the ion becomes a new neutral atom and the neutral atom becomes an ion. As a result, new populations of neutrals are created and their distribution reflects the plasma properties at the region, where charge exchange occurs. Near the heliospheric boundary, the interstellar protons are heated and decelerated and therefore new atoms created by charge exchange at this region have properties different from the original ISNs. The original ISNs are commonly called as the primary population, and the neutrals created by charge exchange from disturbed interstellar ions around the heliopause are called as the secondary population. The secondary atoms have smaller bulk velocity and higher temperature compared to the primary. Inside the heliosphere (i.e. in the region occupied by the solar wind) both primary and secondary ISNs penetrate close to the Sun where they are exposed to the solar gravitation, solar radiation pressure, ionization due to charge exchange with the solar wind protons, and the photoionization. Therefore, inside the heliosphere the spatial and velocity distribution of the interstellar atoms reflect both parameters of the pristine interstellar matter (due to primary component) and 
the plasma properties at the heliospheric boundary (due to secondary component). That is why measurements of the ISNs provide a very important piece of information for heliospheric research.

There are several ways for indirect diagnostic of the ISN atoms distribution in the heliosphere. One of the most well known and widely used method is measuring the backscattered solar Ly $\alpha$ radiation, started at the beginning of the space age (Bertaux \& Blamont 1971; Thomas \& Krassa 1971). Analysis of the intensity and spectral properties of the backscattered Ly $\alpha$ glow in the heliosphere allows to estimate the ISN H parameters far away from the Sun (see e.g. Bertaux et al., 1985; Costa et al., 1999; Lallement et al., 2005; Pryor et al., 2008). Another indirect method is measurements of fluxes of the pick-up ions created from the ISN neutrals $(\mathrm{H}$ and $\mathrm{He})$ due to charge exchange in the heliosphere (Gloeckler et al., 1993). Bzowski et al. (2008) have used the Ulysses data on the pick-up ions to estimate the number density of ISN H atoms at the termination shock.

All indirect methods provide information on the ISN parameters averaged over spatial and/or velocity space (e.g. integrated along line of sight and energy) and do not allow to study the local parameters of atoms inside the heliosphere directly. Therefore, direct measurements of the ISN fluxes could give an important additional information. Direct measurements of the ISN He fluxes were performed before by Ulysses spacecraft, and these data were used for determination of the pristine interstellar parameters (Möbius et al., 2004; Witte 2004 and references therein) because helium atoms penetrate to the heliosphere almost freely due to small charge exchange cross-section. The IBEX mission has been performing extensive direct measurements of the ISN He, H, O, and Ne atoms since 2009 (Möbius et al., 2009). There are numerous studies with analysis and interpretations of the IBEXLo ISN He data with discussion of the ISN velocity and temperature (see e.g. Bzowski et al., 2012; Möbius et al., 2012; Katushkina et al., 2014; McComas et al., 2015; Swaczyna et al., 2019) and the discovery of the secondary ISN He population (Kubiak et al., 2014, 2016, 2019; Bzowski et al., 2017). Also, IBEX for the first time observes the primary and secondary interstellar oxygen atoms (Park et al., 2015, 2016, 2019; Schwadron et al., 2016, Baliukin et al., 2017).

The ISN $\mathrm{H}$ atoms are difficult to detect separately because of low energy and contamination of the signal by the ISN He atoms. First samples of the ISN H data obtained in situ by IBEX-Lo are presented by Saul et al. (2012). The crucial parameter, which determines the hydrogen distribution at $1 \mathrm{au}$, is the dimensionless parameter $\mu$ that is a ratio of the solar radiation pressure repulsive force to the solar gravitational attractive force. Schwadron et al. (2013) have analysed the first $3 \mathrm{yr}$ of IBEX observations of the ISN H fluxes and determined the magnitude of $\mu$ as well as the averaged parameters of the ISN H at the termination shock. Katushkina et al. (2015) have presented the numerical modelling and fitting of the IBEX-Lo ISN H data (for one chosen IBEX orbit in 2009) based on the state-of-art kinetic model of the ISN H distribution in the heliosphere. It was found that the data can be fitted by the model results only with the value of $\mu$ that is much larger than it was thought before. Galli et al. (2019) have published the IBEX-Lo ISN H maps obtained in 2009-2018 (we will discuss and use these data further). Finally, Rahmanifard et al. (2019) have used the available IBEX-Lo ISN H data to determine the solar radiation pressure and the $\mu$ parameter for the almost entire solar cycle 24 .

The main difficulty of the numerical modelling of the ISN H distribution in the heliosphere compared with the ISN He is the significant disturbances of the ISN $\mathrm{H}$ distribution function due to charge exchange at the heliospheric boundary. As a result, the velocity distribution function of the interstellar hydrogen at distances 7090 au from the Sun is not maxwellian (Izmodenov 2001, Izmodenov et al., 2013). To take these effects into account, one needs to use self- consistent kinetic-MHD model of the solar wind/LISM interaction (e.g. Baranov \& Malama, 1993; Pogorelov et al., 2009, 2017; Zank et al., 2013; Izmodenov \& Alexashov 2015, 2020; Opher et al., 2020). In our work of Katushkina et al. (2015), we have applied such a model for the analysis of IBEX-Lo data in 2009. Now we redo the analysis for all available data in 2009-2018. The goals of this work are to use IBEX-Lo ISN H data for (1) the determination of the solar radiation pressure and the hydrogen ionization rate at $1 \mathrm{au}$, (2) deriving the averaged parameters of the secondary ISN $\mathrm{H}$ atoms far away from the Sun and comparison with the results of the heliospheric model, and (3) the analysis of temporal variations of the ISN H fluxes measured by IBEX-Lo in 2009-2018 based on the numerical model.

\section{IBEX-LO ISN H DATA}

IBEX is orbiting the Earth, rotating around its spin axis, and measuring the ISN fluxes in the plane that is perpendicular to the spin axis. The observational time is divided by $7-8$ days orbits. At the beginning of each orbit, the spin axis is directed approximately to the sun and then its direction remains constant during the orbit. So, during each orbit IBEX performs measurements at the fixed plane. This geometry of observations allows to get a complete map of the sky for 6 months. The best season for measurements of the ISN H fluxes is March of each year, when $\mathrm{H}$ atoms move towards IBEX and their relative energy in the spacecraft reference frame is the largest. To improve statistics and increase signal-to-noise ratio the measured counts are accumulated during each orbit in $6^{\circ}$ bins of spin angle. The spin angle is counting in the observational plane and this is an analogue of the ecliptic latitude because the observational plane is almost perpendicular to the ecliptic plane. The main difficulty of data processing is how to separate the ISN H signal in the raw data from different species. Incoming atoms of $\mathrm{H}$ and $\mathrm{O}$ are converted to negative ions at the instrument's conversion surface and ions energy distribution is just a little bit shifted to lower energy compared to original energy distribution of atoms. In contrast, the noble gases such as ISN He and Ne produce sputtered ions $\left(\mathrm{H}^{-}, \mathrm{O}^{-}, \mathrm{C}^{-}\right)$with a broad energy distribution for all energies below the energy of incoming atom (Möbius et al., 2012).

Galli et al. (2019) describe in details the raw IBEX-Lo data (count rate of $\mathrm{H}^{-}$) and propose several methods to derive the ISN $\mathrm{H}$ fluxes. These methods allow to separate the signal of the ISN H from other species (mostly from the ISN He). In this work, we will use the data obtained in Galli et al. (2019) using 'H3 inflight' approach that was chosen as a default method in their paper. Originally, the data are presented in the 'orbit-spin' format, where the ISN H flux is a function of IBEX orbit's number and spin angle (spin angles in the range of $180^{\circ}-360^{\circ}$ correspond to the ram direction towards the ISN flow). But, it is possible to transform the fluxes in the format of ecliptic maps using a procedure of spatial re-binning. In this work for comparison of the IBEX data with the model results, we preferably use the original orbit-spin format of the data, but sometimes for better representation we use the ecliptic format.

Fig. 1 shows an example of the data obtained by IBEX-Lo sensor in 2009 at the energy bins $1(11-21 \mathrm{eV})$ and $2(20-41 \mathrm{eV})$ together with maps of the relative uncertainties. The apparent absence of ISN $\mathrm{H}$ fluxes at the left part of the map is due to the very intense signal of ISN He atoms, which are measured by IBEX earlier than the ISN $\mathrm{H}$ during each year. It is seen that the fluxes in energy bin 1 are much larger than in energy bin 2. On the contrary, as it was shown by Katushkina et al. (2015) the state-of-art kinetic model of the ISN $\mathrm{H}$ distribution in the heliosphere predicts about the same fluxes at energy bins 1 and 2. We will discuss this problem is Section 7. To 

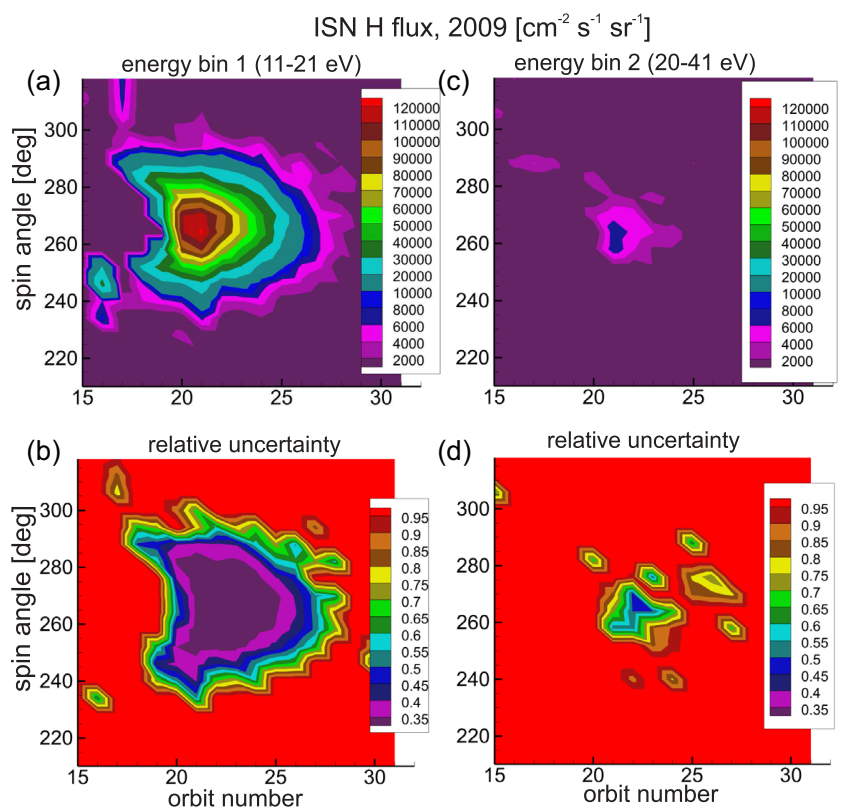

Figure 1. The ISN H fluxes measured in 2009 by IBEX-Lo in two first energy bins. These data were originally processed and published by Galli et al. (2019). Plots (a) and (c) show maps of the fluxes, plots (b) and (d) show maps of relative uncertainties that is the ratio of uncertainty to the flux for each direction. Note that the red pixels in the relative uncertainty maps correspond to the directions where the derived ISN H signal is not significant against background and other signals (in particular the ISN He signal). Maps are presented in 'orbit-spin' format.

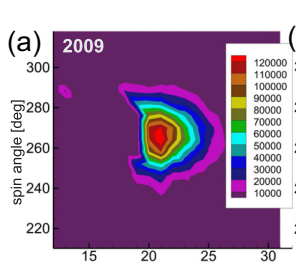

ISN H fluxes for bin1+bin2, [cm $\mathrm{cm}^{-2} \mathrm{sr}^{-1}$
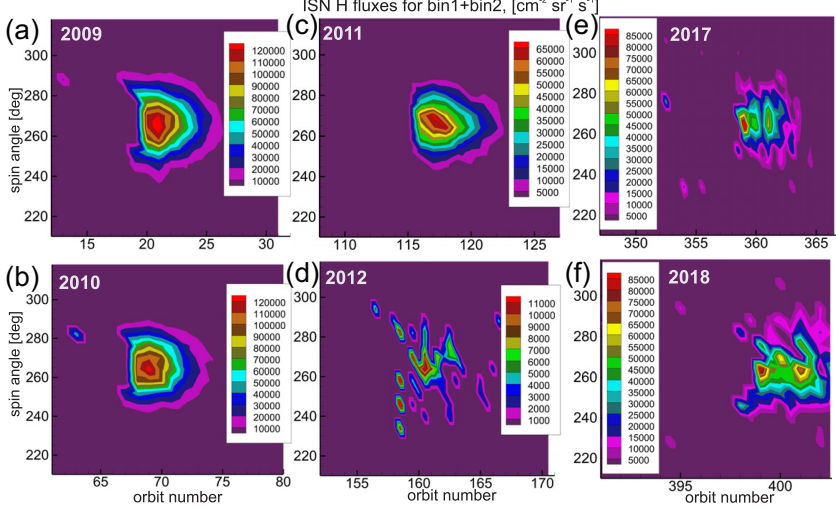

Figure 2. IBEX-Lo data: maps of the ISN H fluxes measured in the energy bins 1 and 2 together. The data are presented in 2009-2011 and 2017-2018 for those direction where relative uncertainty is less than 0.9. Maps are shown in 'orbit-spin' format. Resolution of spin angle is $6^{\circ}$, resolution in orbit's number is 1 before 2012 and 0.5 after 2012 .

avoid any possible confusions with separation of incoming atoms into energy bins we decide to consider a sum of the fluxes measured in the energy bins 1 and 2, i.e. all ISN H atoms together.

Fig. 2 presents maps of the ISN H fluxes measured by IBEX-Lo in 2009-2012 and 2017-2018 in energy bins 1 and 2 together. Here, we keep the data for those directions where the relative uncertainty is less than 0.9. We omit maps obtained in 2013-2016 because as it is shown by Galli et al. (2019) the measured fluxes during these years near the solar maximum are small and statistics is not enough to thrust these data. In Section 7, we use just several data points extracted from the maps in 2013-2016 with appropriate statistic. It should be noted that

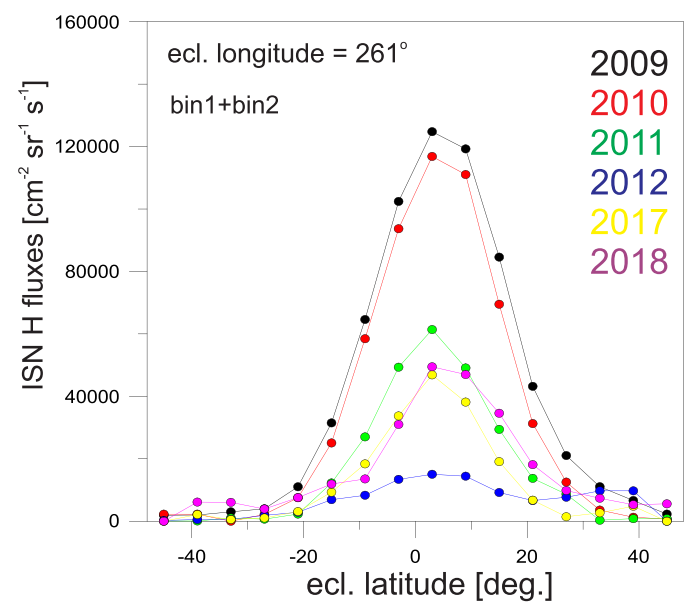

Figure 3. IBEX-Lo data: ISN H fluxes measured in the energy bins 1 and 2 together for different years. Ecliptic longitude of the line of sight is $261^{\circ}$, and data are presented for slices over ecliptic latitude.

since 2012 each IBEX's orbit starts to be divided by two separate arcs with different directions of spin axis. Therefore, in 2009-2011 temporal resolution ( $x$-axis on the maps in Fig. 2 ) is one orbit (about $9 \mathrm{~d}$ ), while in 2012 and after the resolution is a half of the orbit. That is why accumulation time is less after change of the orbits duration and therefore statistic in 2012, 2017, and 2018 is worse compared to 2009-2011 as it is seen from Fig. 2. We will use these maps further for comparison and fitting of the data with the model results.

Temporal variations of the measured fluxes are presented in Fig. 3. This figure shows the ISN $\mathrm{H}$ fluxes as functions of ecliptic latitude for the fixed ecliptic longitude of the line-of-sight $\left(261^{\circ}\right)$. This direction is chosen because in 2009 it corresponds to the maximum ISN H fluxes. It is seen that the fluxes are the largest at the solar minimum 2009-2010, then they decrease and reach the lowest magnitude in 2012 (the fluxes are even smaller in 2013-2015, but statistic is not enough and we do not consider maps in 2013-2016), and then start to increase again in 2017-2018. This behaviour is coupled to the solar cycle because at the solar maximum both the solar radiation pressure and the ionization rate are high, hence the ISN $\mathrm{H}$ atoms are swept out further from the Sun and ionized a lot and their fluxes at 1 au are extremely low.

\section{MODEL DESCRIPTION}

The kinetic model of the ISN H distribution in the heliosphere that is used for analysis of IBEX-Lo data was described in details by Izmodenov et al. (2013) and Katushkina et al. (2015). Here, we briefly summarize the main aspects.

The spatial and velocity distribution of the ISN H atoms in the heliosphere is described by kinetic equation:

$$
\begin{aligned}
& \frac{\partial f(\boldsymbol{r}, \boldsymbol{w}, t)}{\partial t}+\boldsymbol{w} \cdot \frac{\partial f(\boldsymbol{r}, \boldsymbol{w}, t)}{\partial \boldsymbol{r}}+ \\
& \frac{\boldsymbol{F}\left(\mathbf{r}, t, \lambda, w_{r}\right)}{m_{\mathrm{H}}} \cdot \frac{\partial f(\boldsymbol{r}, \boldsymbol{w}, t)}{\partial \boldsymbol{w}}=-\beta(r, t, \lambda) \cdot f(\boldsymbol{r}, \boldsymbol{w}, t) .
\end{aligned}
$$

Here, $f(\boldsymbol{r}, \boldsymbol{w}, t)$ is the velocity distribution function of $\mathrm{H}$ atoms, $\boldsymbol{w}$ is the individual velocity of an $\mathrm{H}$ atom, and $m_{\mathrm{H}}$ is the mass of an $\mathrm{H}$ atom. $\boldsymbol{F}$ is the force acting on each atom in the heliosphere that is a combination of the solar gravitational attractive force $\left(\boldsymbol{F}_{g}\right)$ and the solar radiative repulsive force $\left(\boldsymbol{F}_{\text {rad }}\right)$. Both forces are proportional to $1 / r^{2}$ ( $r$ is the heliocentric distance), and it is convenient to consider 
the dimensionless parameter $\mu=\left|\boldsymbol{F}_{\text {rad }}\right| /\left|\boldsymbol{F}_{g}\right|$. Then,

$$
\begin{aligned}
\boldsymbol{F} & =\boldsymbol{F}_{g}+\boldsymbol{F}_{\mathrm{rad}}=\left(1-\mu\left(t, \lambda, w_{\mathrm{r}}\right)\right) \boldsymbol{F}_{g}= \\
& =-m_{\mathrm{H}} \frac{\left(1-\mu\left(t, \lambda, w_{\mathrm{r}}\right) G M_{\mathrm{s}}\right.}{r^{2}} \cdot \frac{\boldsymbol{r}}{r},
\end{aligned}
$$

where $G$ is the gravitational constant and $M_{\mathrm{s}}$ is the mass of the Sun. In general, the parameter $\mu$ depends on the time $(t)$, heliolatitude $(\lambda)$, and the radial component of the atom's velocity $\left(w_{\mathrm{r}}\right)$.

The right-hand side of the kinetic equation (1) represents the losses of $\mathrm{H}$ atoms due to charge exchange with the solar wind protons and the photoionization. Note that we consider here only the interstellar populations of $\mathrm{H}$ atoms and do not consider the heliospheric populations created by charge exchange inside the heliosphere (part of them are known as ENAs). $\beta$ is the total ionization rate that is a sum of the charge exchange ionization rate $\beta_{\text {ex }}$ and the photoionization rate $\beta_{\mathrm{ph}}$. Inside the heliospheric termination shock both of these terms are proportional to $1 / r^{2}$ (although for the solar wind mass flux and the charge exchange ionization rate this may not be strictly correct due to an influence of the pick-up ions and corresponding deceleration of the solar wind, but this effect is negligible, especially because most of the atoms are ionized close to the Sun), therefore the total ionization rate can be adjusted to $1 \mathrm{au}$ :

$\beta(r, t, \lambda)=\left(\beta_{e x, E}(t, \lambda)+\beta_{p h, E}(t, \lambda)\right)\left(\frac{r_{E}}{r}\right)^{2}=\beta_{E}(t, \lambda)\left(\frac{r_{E}}{r}\right)^{2}$,

where $r_{E}=1 \mathrm{au}$, subscript $E$ indicates that the ionization rates are taken at $1 \mathrm{au}$. The total ionization rate $\beta_{E}$ depends on time and heliolatitude due to temporal and latitudinal variations of the solar wind and the solar radiation.

The boundary conditions for equation (1) are taken at 70 au from the Sun (this sphere is located inside the termination shock). By default, the ISN H velocity distribution function at 70 au is a sum of the distribution functions of the primary and secondary interstellar atoms, and each of them is represented by a 3D normal distribution, which takes into account all zero (number density), first (averaged velocity), and second (tensor of kinetic temperatures at different directions) moments of the distribution function (see details in Katushkina et al., 2015). The moments at sphere with radius 70 au depend on two angles and are obtained from the results of the global kinetic-MHD model of the interaction between the solar wind and the interstellar matter proposed recently by Izmodenov \& Alexashov (2020). Hereafter, we will refer to this model as IA2020. The obtained $\mathrm{H}$ parameters at $70 \mathrm{au}$ are different from the original parameters of the interstellar wind due to filtration of the primary component of $\mathrm{H}$ atoms at the heliospheric interface and creation of the secondary component near the heliospheric boundary. Izmodenov \& Alexashov (2020) have reported that the number density of $\mathrm{H}$ atoms at $70 \mathrm{au}$ in the upwind direction obtained in the model is $0.097 \mathrm{~cm}^{-3}$ that agrees with the observational constrains (Geiss et al. 2006; Bzowski et al. 2008); and the $\mathrm{H}$ deflection relative to the He flow obtained in the model is $3.5^{\circ}$ that agrees with a number of $4^{\circ}$ obtained by Lallement et al. $(2005,2010)$ from analysis of SOHO/SWAN data on the backscattered Ly $\alpha$ radiation.

We use the stationary boundary conditions at $70 \mathrm{au}$ because variations of the ISM H parameters during the solar cycle at such distance from the Sun are within 10 per cent that is much smaller than uncertainties in the IBEX-Lo data. For some specific test calculations, we use simple maxwellian boundary conditions for the velocity distribution function of the primary and secondary ISN $\mathrm{H}$ atoms at $70 \mathrm{au}$ without angular dependence of its moments. The following LISM parameters are used in the global IA2020 model: the number density of protons is $n_{\mathrm{p} \text {, LISM }}=0.04 \mathrm{~cm}^{-3}$; the number density of $\mathrm{H}$ atoms is $n_{\mathrm{H}, \mathrm{LISM}}=0.14 \mathrm{~cm}^{-3}$; the velocity of the interstellar wind is $V_{\text {LISM }}=26.4 \mathrm{~km} \mathrm{~s}^{-1}$ and its direction is derived from the Ulysses ISN He data analysis reported by Witte et al. (2004), i.e. the ecliptic (J2000) longitude is $75.4^{\circ}$ and the latitude is $-5.2^{\circ}$ (this velocity vector is in agreement with results derived from IBEX data, see e.g. McComas et al. 2015); the interstellar temperature is $T_{\text {LISM }}=6530 \mathrm{~K}$ that corresponds to the results of Witte et al. (2004) and is a little bit smaller than the temperature of the interstellar He flow derived by McComas et al. (2015); the interstellar magnetic field vector belongs to the hydrogen deflection plane (Lallement et al., 2005, 2010), its magnitude is $B_{\text {LISM }}=3.75 \mu \mathrm{G}$ and the angle between $\mathbf{B}_{\text {LISM }}$ and $\mathbf{V}_{\text {LISM }}$ is $60^{\circ}$. Izmodenov \& Alexashov (2020) have obtained such direction and magnitude of the pristine local interstellar magnetic field based on analysis of the interstellar magnetic field components at the heliopause measured by magnetometer instruments onboard Voyager 1 and 2 spacecraft. IA2020 model provides a good agreement with Voyager 1 and 2 data before and after their crossing the heliopause.

By default, the input parameters of the model $\mu\left(t, \lambda, w_{\mathrm{r}}\right)$ and $\beta(t$, $\lambda)$ are calculated based on observational data. Namely, $\mu\left(t, \lambda, w_{\mathrm{r}}\right) \mathrm{de}-$ pends on the solar Ly $\alpha$ irradiance, that is known from LISIRD/LASP data base (Machol et al., 2019), its dependence of heliolatitude and radial velocity is modelled by Kowalska-Leszczynska et al. (2018, 2020). The charge exchange ionization rate in the ecliptic plane can be calculated based on the solar wind parameters measured at the Earth orbit and summarized by OMNI data base. Photoionization rate can be calculated from the solar extreme ultraviolet (EUV) radiation flux. Note that the solar EUV flux certainly correlates with the solar radiation pressure therefore the photoionization rate and magnitudes of $\mu$ are related to each other. But, as shown by, e.g. Sokół et al. (2019) the photoionization rate of $\mathrm{H}$ atoms is about one order of magnitudes less than the charge exchange ionization rate. Therefore, the total ionization rate $\beta$ and $\mu$ can be considered as independent model parameters. Heliolatitudinal variations of the total ionization rate are taken from the results of analysis of SOHO/SWAN data on Ly $\alpha$ intensity maps, which allows to obtain temporal and heliolatitudinal variations of the ionization rate (Quemerais et al., 2006; Lallement et al., 2010; Koutroumpa et al., 2019). In this work, we use the IBEX-Lo ISN $H$ fluxes to derive the magnitude of $\mu$ and $\beta_{E}$ independently by fitting the IBEX-Lo data with the model results (see Section 5).

To calculate the ISN H fluxes in IBEX observational geometry, one needs to integrate the $\mathrm{H}$ velocity distribution function over energy within corresponding energy range, take into account integration over collimator and averaging over $6^{\circ}$ angular bin, as it is done for the data. Appendix A provides formulas for calculations of the ISN $\mathrm{H}$ fluxes used for the comparison with the data.

\section{EFFECT OF MAGNITUDE OF $\mu$ AND $\beta$ FOR THE ISN H MAP}

In this section, we show how the magnitude of the solar radiation pressure $(\mu)$ and the ionization rate $(\beta)$ influence the ISN H map. For the model calculations, we use the IBEX-Lo observational geometry corresponding to 2009. To separate the effects we consider the stationary version of our numerical model with constant $\mu$ and $\beta$. Fig. 4 presents the IBEX-Lo maps calculated for 2009 in orbit-spin format for different magnitudes of $\mu$ and $\beta$. From the top row of plots (a)-(c), it is seen that increase of $\mu$ results in decrease of the ISN $\mathrm{H}$ fluxes and shift of maximum to the right part of the map. This is explained by changing of $\mathrm{H}$ atoms trajectories with increasing of the 

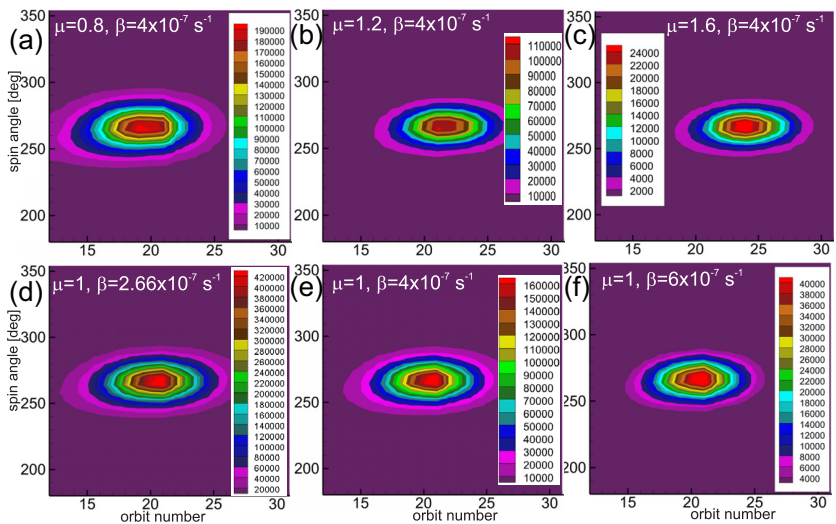

Figure 4. Model results: maps of ISN H fluxes obtained in the frame of the stationary model with constant $\mu$ and $\beta$. Fluxes are calculated for energy bins 1 and 2 together. Maps are calculated using geometry of IBEX-Lo observations. Fluxes are given in $\left[\mathrm{cm}^{-2} \mathrm{~s}^{-1} \mathrm{sr}^{-1}\right]$.

radiation pressure. Note that the shape of the map is about the same for all variants. Plots (d)-(f) in Fig. 4 shows an influence of $\beta$. It is seen that magnitude of $\beta$ does not influence the shape of the map and position of the maximum, but magnitudes of the fluxes are changed dramatically. Increase of $\beta$ by a factor of 2.25 leads to decrease of the fluxes by a factor of 10.5 . Thus, position of the maximum fluxes for each map reflects magnitude of $\mu$, while $\beta$ is responsible only for scaling of the maps.

\section{STEP 1: FITTING OF THE DATA TO SEARCH FOR $\mu_{0}$ AND $\beta_{0}$}

To fit the IBEX-Lo ISN H data presented in Section 2, we use our kinetic model described above and vary parameters $\mu$ and $\beta$ to minimize $\chi^{2}$. Namely, for each year we have the IBEX-Lo map of the $\mathrm{H}$ fluxes with relative errors less than 0.9. We use the model of $\mathrm{H}$ distribution with the following parameters:

(i) $\mu\left(t, \lambda, w_{\mathrm{r}}\right)=\mu_{0} \cdot \frac{F_{\mathrm{KL}}\left(t, \lambda, w_{\mathrm{r}}\right)}{F_{\mathrm{KL}}\left(t, \lambda, w_{\mathrm{r}}=0\right)}$, where $F_{\mathrm{KL}}\left(t, \lambda, w_{\mathrm{r}}\right)$ is the function proposed by Kowalska-Leszczynska et al. (2018) and corrected by Kowalska-Leszczynska et al. (2020) in accordance with new version of the LISIRD/LASP data set of the solar Ly $\alpha$ irradiance.

(ii) $\beta_{\mathrm{E}}(t, \lambda)=\beta_{0} \cdot \frac{\beta_{\mathrm{E}, \text { model }}(\lambda)}{\beta_{\mathrm{E} \text {,model }}\left(\lambda=0^{\circ}\right)}$, where $\beta_{\mathrm{E} \text {, model }}(\lambda)$ is the total ionization rate described above (obtained from OMNI and SOHO/SWAN data) averaged over considered time period for one IBEX-Lo map.

Thus, we keep the dependence of $\mu$ on heliolatitude and the atom's radial velocity and dependence of $\beta$ on heliolatitude the same as it was used in the model by default. Then, we vary parameters $\mu_{0}$ and $\beta_{0}$ in wide ranges, namely, $\mu_{0}$ was varied from 0.6 to 1.6 with a step of 0.01 and $\beta_{0}$ was varied from $2 \times 10^{-7}$ to $8 \times 10^{-7} \mathrm{~s}^{-1}$ with a step of $0.1 \times 10^{-7} \mathrm{~s}^{-1}$. For each set of $\mu_{0}$ and $\beta_{0}$, we run the model and calculate a metric of reduced $\widehat{\chi}^{2}$ for each map:

$\hat{\chi}^{2}\left(\mu_{0}, \beta_{0}\right)=\frac{1}{N-2} \sum_{i=1}^{N}\left(\frac{F_{\text {data }, i}-F_{\text {model }, i}\left(\mu_{0}, \beta_{0}\right)}{\sigma_{\text {data }, i}}\right)^{2}$.

Here, $\mathrm{N}$ is the number of the considered data points for one map, $F_{\text {data, } i}$ and $F_{\text {model, } i}$ are the ISN $H$ fluxes of the data and the model results, correspondingly, $\sigma_{\text {data }, i}$ is the uncertainties of the data. Note that we use only those directions where the fluxes in the data are non-zero and the relative uncertainties are less than 0.9 , i.e. in the model we also do not consider the left part of the map, which is contaminated by signal from helium. By the least-squares method we find the 'best-fitting' values of $\mu_{0}$ and $\beta_{0}$, which provide the minimum of $\hat{\chi}^{2}$ separately for each year.

We do this for three different versions of the numerical model in order to investigate how the results are sensitive to the model parameters. Namely, we use the following:

(i) Model 1 is the initial 3D quasi-stationary model with nonmaxwellian boundary conditions at $70 \mathrm{au}$. This model is described above.

(ii) Model 2 is the same as the Model 1, but without heliolatitudinal variations of the ionization rate, i.e. for this case $\beta_{E}$ depends only on time;

(iii) Model 3 is the same as the Model 1 but with simple maxwellian boundary conditions at 70 au for primary and secondary interstellar atoms; parameters of the maxwellian distributions were taken from the initial IA2020 model at 70 au in the upwind direction, i.e. direction towards the interstellar wind flow (the parameters are listed in Table 1).

The results of the fitting are presented in Fig. 5. Plots of $\hat{\chi}^{2}$ and calculations of the uncertainties for the found parameters are presented in Appendix B. Uncertainties are presented only for the results of Model 1 to make the plot more readable. Uncertainties for other models are the same order of magnitude. Note that for most years the obtained values of the minimal reduced $\hat{\chi}^{2}$ are less than 1 that is generally considered to be an indication of overestimation of the data uncertainties.

First of all let us consider the results obtained with the Model 1 and compare them with the initial values of $\mu_{0}$ and $\beta_{0}$ presented in Fig. 5(a) and (b). It is seen that the obtained best-fitting values of $\mu_{0}$ agree within the uncertainties with the initial ones for all years except 2009. In 2009, the IBEX-Lo data are fitted with $\mu_{0}=0.98$, while the model of Kowalska-Leszczynska et al. (2020) provides $\mu_{0}=0.84$ for this period of time and $w_{\mathrm{r}}=0$. Note, that previously Katushkina et al. (2015) have obtained $\mu_{0}=1.29$ based on analysis of IBEX-Lo data for one orbit in 2009. This value is much larger than found here. This is explained by the following: Katushkina et al. (2015) have performed a fitting of the data for both first and second energy bins separately, and large $\mu_{0}$ was obtained because of unexpected ratio of fluxes in energy bins 1 and 2 seen in the data, which cannot be explained by the model with smaller $\mu$. Now we consider a sum of

Table 1. Parameters of the primary and secondary interstellar $\mathrm{H}$ atoms derived at 70 au in the upwind direction from the results of the global IA2020 heliospheric model.

\begin{tabular}{lccccccc}
\hline & $n_{\mathrm{H}} / n_{\mathrm{H}, \text { LISM }}$ & $|\mathbf{V}| / V_{\mathrm{LISM}}$ & ecl. lon. & ecl. lat. & $T_{x}$ & $T_{y}$ & $T_{z}$ \\
\hline Primary & 0.22 & 1.038 & $74.58^{\circ}$ & $-6.47^{\circ}$ & $6755 \mathrm{~K}$ & $6374 \mathrm{~K}$ & $5903 \mathrm{~K}$ \\
Secondary & 0.473 & 0.678 & $71.4^{\circ}$ & $-6.62^{\circ}$ & $15496 \mathrm{~K}$ & $12207 \mathrm{~K}$ & $12122 \mathrm{~K}$ \\
\hline
\end{tabular}

Note. $n_{\mathrm{H}, \mathrm{LISM}}=0.14 \mathrm{~cm}^{-3}, V_{\mathrm{LISM}}=26.4 \mathrm{~km} \mathrm{~s}^{-1}$ (Izmodenov \& Alexashov, 2020); The coordinate system $(x, y, z)$ in the global model is the following: $z$-axis towards opposite upwind direction, plane $(z, x)$ coincides with $\left(\mathbf{V}_{\text {LISM }}\right.$, $\mathbf{B}_{\text {LISM }}$ )-plane, $x$-axis is perpendicular to $z$ and $B_{\text {LISM, } x}<0, y$-axis completes a right-hand coordinate system. 

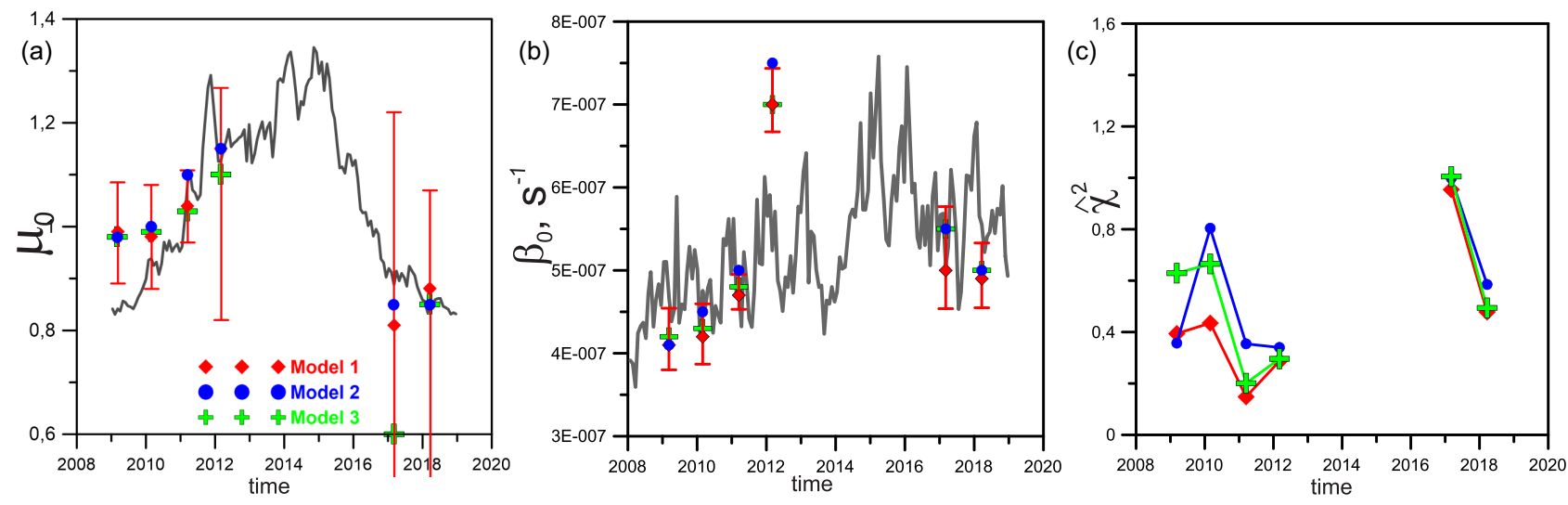

Figure 5. Results of the fitting procedure and comparison with the data known before. Plots (a) and (b) show values of $\mu_{0}$ and $\beta_{0}$ as functions of time. The grey lines correspond to $\mu_{0}(t)$ provided by the model of Kowalska-Leszczynska et al. (2020) with $w_{\mathrm{r}}=0$, and the total ionization rate at 1 au calculated based on measurements of the solar wind and solar radiation parameters (known from OMNI 2 and LISIRD/LASP data bases). The lines with different symbols and colors show the 'best-fitting' values of $\mu_{0}$ and $\beta_{0}$ with uncertainties obtained by three models described in the text. The obtained uncertainties are shown only for the results of Model 1 to make a figure not too much busy, uncertainties for other models are the same order of magnitude. Plot (c) shows the minimum of reduced $\hat{\chi}^{2}$ obtained for each year using three models.

the fluxes in two bins that seems to be safer due to possible technical issues with determination of incoming atom's energy. This allows us to get a smaller and more realistic magnitudes of $\mu_{0}$ in 2009. Note that the magnitude of $\hat{\chi}^{2}$ obtained by Katushkina et al. (2015) was much larger than that obtained here (see Plot c in Fig. 5). Rahmanifard et al. (2019) have obtained $\mu_{0} \approx 1$ for 2009 that is close to our results. Let us note that 2009 is a deep solar minimum with very low solar ionization and solar wind fluxes. Therefore, an amount of $\mathrm{H}$ atoms in the vicinity of the Sun in 2009 is the largest compared to other years. It is commonly assumed in the numerical models that the solar radiation pressure force decreases with distance from the Sun as $\sim 1 / r^{2}$. This assumption is valid if the $\mathrm{H}$ atoms in the heliosphere are optically thin for Ly $\alpha$ photons. However, generally speaking, this is not the case of the heliosphere, where multiply scattering of photons is important as it is shown by Quémerais (2000). The more atoms in the heliosphere, the greater the effect of multiple scattering, so this is especially important during deep solar minimum of 2009. In the case of optically thick matter the solar Ly $\alpha$ flux and the solar radiation pressure as well should decrease with distance from the Sun slower than $1 / r^{2}$ (Fayock et al., 2015). This could be an explanation of larger magnitude of $\mu_{0}$ obtained from the IBEX-Lo data in 2009. In 2017, the obtained best-fitting $\mu_{0}=0.81$ is a little bit smaller than the known one 0.9. However, the error bars for the found $\mu_{0}$ are quite large after 2012 due to uncertainties in the data.

The magnitudes of $\beta_{0}$ obtained by the fitting procedure in 2009 2011 and 2017-2018 are a little bit smaller (but within the error bars) than the averaged level of known ones. This means that the data show that amount of $\mathrm{H}$ atoms at 1 au is larger than it is in the model results. This may be caused by underestimation of the LISM number density of $\mathrm{H}$ atoms in the model or with uncertainties of IBEX-Lo calibration factor. Significant difference between the found $\beta_{0}$ and the known one occurs in 2012. In 2012 the best-fitting $\beta_{0}=7 \times 10^{-7} \mathrm{~s}^{-1}$, while the value in the ecliptic plane obtained based on data on the ionization rate at 1 au is about $\beta_{0}=5.78 \times 10^{-7} \mathrm{~s}^{-1}$ (see Fig. 5b). We discuss the obtained difference further in the paper in Section 8.

Results of the Model 2 are very close to the results of the Model 1 that means that the latitudinal variations of the ionization rate do not influence the position of the maximum fluxes as well as magnitude of the fluxes (at least at considered parts of the maps, which are not contaminated by signal from the ISN He atoms). The Model 3 gives approximately the same results as the Models 1 and 2 except one point in 2017, when the best-fitting $\mu_{0}$ obtained by the Model 3 is significantly less than the corresponding ones obtained by the Models 1 and 2.

Thus, it is seen that results of different models are close to each other, hence the determination of $\mu_{0}$ and $\beta_{0}$ from the IBEX-Lo ISN H maps can be considered as the model-independent procedure. From Fig. 5(c), it is seen that in 2009-2011 the minimal $\hat{\chi}^{2}$ calculated for the Model 3 is larger than for Models 1 and 2, while in 2012 and 2017-2018 all models give about the same $\hat{\chi}^{2}$.

\section{STEP 2: FITTING OF THE DATA TO SEARCH FOR ISN H PARAMETERS AT 70 AU FROM THE SUN}

At the previous step to fit the data, we use the kinetic model with parameters of ISN $\mathrm{H}$ at $70 \mathrm{au}$ taken from results of the global self-consistent kinetic-MHD model of the heliosphere (IA2020). However, these hydrogen parameters at 70 au certainly depend on the outer boundary conditions stated in the undisturbed interstellar medium at the global model as well as on the physical precesses at the heliospheric boundary. Generally speaking, the LISM parameters such as the protons and $\mathrm{H}$ atoms number densities and magnetic field are not known precisely. Therefore, it is interesting to determine the ISN H parameters at 70 au from the Sun by fitting the IBEX-Lo data and explore, are they consistent with the results of the global model or not.

To do this, we assume that the velocity distribution function of both primary and secondary interstellar atoms at 70 au is maxwellian (with three kinetic temperatures) and parameters of these maxwellian functions are the same at the whole boundary sphere. The distribution function is the following:

$$
\begin{aligned}
f_{\mathrm{H}, i}(\mathbf{w})= & \frac{n_{\mathrm{H}, i}}{\pi \sqrt{\pi} D} \times \exp \left(-\frac{\left(V_{x, i}-w_{x}\right)^{2}}{c_{x, i}^{2}}-\frac{\left(V_{y, i}-w_{y}\right)^{2}}{c_{y, i}^{2}}\right. \\
& \left.-\frac{\left(V_{z, i}-w_{z}\right)^{2}}{c_{z, i}^{2}}\right),
\end{aligned}
$$

where $D=c_{x, i} \cdot c_{y, i} \cdot c_{z, i,} c_{a, i}=\sqrt{2 T_{a, i} k_{b} / m_{\mathrm{H}}}, a=(x, y, z), i=(1$, 2 ) is the population's number ( 1 for primary interstellar atoms and 2 
Table 2. ISN H parameters derived by Levenberg-Marquardt method from IBEX-Lo map in 2009.

\begin{tabular}{lccc}
\hline & Set 1 & Set 2 & Set 3 \\
& $\mu_{0}=0.99$ & $\mu_{0}=0.84$ & $\mu_{0}=0.99$ \\
& $\beta_{0}=4.1 \times 10^{-7} \mathrm{~s}^{-1}$ & $\beta_{0}=4.67 \times 10^{-7} \mathrm{~s}^{-1}$ & $\beta_{0}=4.67 \times 10^{-7} \mathrm{~s}^{-1}$ \\
\hline$n_{\mathrm{H}, 1} / n_{\mathrm{H}, \text { LISM }}$ & $0.36 \pm 0.23$ & $0.356 \pm 3.65$ & $0.52 \pm 0.34$ \\
$n_{\mathrm{H}, 2} / n_{\mathrm{H}, \text { LISM }}$ & $0.20 \pm 0.04$ & $0.368 \pm 0.07$ & $0.27 \pm 0.05$ \\
$\left|\mathbf{V}_{2}\right| / V_{\text {LISM }}$ & $0.82 \pm 0.33$ & $2.85 \pm 24.52$ & $0.805 \pm 0.335$ \\
ecl. lon. & $70.1 \pm 5.5$ & $82.3 \pm 91.2$ & $71.4 \pm 4.9$ \\
ecl. lat. & $-9.4 \pm 4.9$ & $-6.19 \pm 5.5$ & $-9.53 \pm 5.0$ \\
$\alpha_{\text {defl }}^{\circ}$ & $5.26_{-5.2}^{+8.7}$ & $6.98_{-6.6}^{+91.1}$ & $5.87_{-5.8}^{+6.9}$ \\
$T_{x, 2}(\mathrm{~K})$ & $15584 \pm 3838$ & $25318 \pm 12067$ & $15112 \pm 3656$ \\
$T_{y, 2}(\mathrm{~K})$ & $14180 \pm 3841$ & $21423 \pm 10435$ & $13647 \pm 3631$ \\
$T_{z, 2}(\mathrm{~K})$ & $24257 \pm 15292$ & $417871 \pm 5563045$ & $25841 \pm 15542$ \\
$\hat{\chi}^{2}$ & 0.178 & 0.207 & 0.19 \\
\hline
\end{tabular}

for secondary interstellar atoms), $k_{\mathrm{b}}$ is the Boltzmann constant; $n_{\mathrm{H}}$ is the $\mathrm{H}$ number density, $\boldsymbol{V}$ is the averaged velocity vector.

Velocity and temperatures of the original interstellar wind are well known from measurements of the interstellar helium flow by Ulysses and IBEX (Möbius et al., 2004; McComas et al., 2015). Parameters of the primary population of interstellar hydrogen are slightly different from the interstellar wind's parameter due to filtration of the heliospheric interface that is known from the results of the heliospheric model. Therefore, we have kept the average velocity vector and three kinetic temperatures of the primary interstellar atoms obtained at $70 \mathrm{au}$ in the upwind direction using the global IA2020 model (see first row in Table 1).

Thus, we have eight remaining parameters: $n_{H, 1}, n_{H, 2}, V_{2}, T_{x, 2}$, $T_{y, 2}, T_{z, 2}$, which should be obtained by fitting of the data. In such a case of large number of parameters, the widely used non-linear method to search for minimum of $\chi^{2}$ is the Levenberg-Marquardt method described in Numerical Recipes (Press et al., 1986). We apply this method for the IBEX-Lo ISN H map (sum of the fluxes for energy bins 1 and 2 as before) measured in 2009 because for this map the measured fluxes are the largest and signal-to-noise ratio is better compared with other years, so the fitting procedure is safer. We run the Levenberg-Marquardt method using the 3D quasi-stationary version of kinetic model for $\mathrm{H}$ distribution and three sets of $\mu_{0}$ and $\beta_{0}$ :

(i) Set 1: $\mu_{0}=0.9899, \beta_{0}=4.1 \times 10^{-7} \mathrm{~s}^{-1}-$ these magnitudes are obtained for 2009 by the fitting procedure described above in Section 5;

(ii) Set 2: $\mu_{0}=0.84, \beta_{0}=4.67 \times 10^{-7} \mathrm{~s}^{-1}-$ these are default values known before that are shown in Fig. 5 by grey colour.

(iii) Set 3: $\mu_{0}=0.9899, \beta_{0}=4.67 \times 10^{-7} \mathrm{~s}^{-1}-$ intermediate set with $\mu_{0}$ from Set 1 and $\beta_{0}$ from Set 2 .

The derived ISN H parameters with corresponding uncertainties and minimum $\hat{\chi}^{2}$ are presented in Table 2. Note that uncertainties of the found parameters are calculated using recipe of LevenbergMarquardt and they are quite large due to large uncertainties of the IBEX-Lo data. It is seen that for Set 1 the found parameters are adequate and very close to the parameters obtained in the frame of the global IA2020 heliospheric model (see Table 1 for comparison). $\alpha_{\text {defl }}$ presented in Table 2 is the deflection angle between the averaged velocity vector of the secondary interstellar atoms at $70 \mathrm{au}$ and original interstellar wind direction. IA2020 model provides $\alpha_{\text {defl }}=$ $4.22^{\circ}$. The model with parameters of Set 1 provides a little bit larger deflection, but with significant error bars. $T_{z}$ component of temperature found from IBEX-Lo data is larger than that provided by the global model, but uncertainty for this component is very large, and this means that $T_{z}$ cannot be determined precisely from the presented data. Note that the obtained minimum $\hat{\chi}^{2}=0.178$ is about two times smaller than found before for 2009 in Section 5 by fitting of the data with the initial Model 1. The ISN H parameters obtained with Set 2 (with $\mu_{0}=0.84$ ) from the IBEX-Lo map seemed unrealistic. The found velocity (both magnitude and direction) and temperature $T_{z}$ cannot be physically explained. At the same time, the parameters obtained with Set 3 are very close to those obtained with Set 1 (only number densities are larger in the results obtained with Set 3 that is obvious because ionization rate is higher). This means that magnitude of $\mu_{0}$ is extremely important for the IBEX-Lo ISN map and $\mu_{0}=0.84$ is not consistent with the data, while the new one $\mu_{0}=0.9899$ is consistent and provide realistic parameters of the secondary interstellar $\mathrm{H}$ at 70 au from the Sun, which agree well with the IA2020 model results.

\subsection{Step 3: obtaining of $\mu_{0}$ and $\beta_{0}$ with found ISN H parameters}

Then, we need to check if the found parameters of the secondary interstellar atoms are suitable for other years. To do this, we keep the ISN $\mathrm{H}$ distribution at $70 \mathrm{au}$ in the form of two maxwellian functions. Parameters (averaged velocity and temperatures) of the primary component are taken from results of the global heliospheric model in the upwind direction, while the number densities of both primary and secondary atoms, velocity and temperature of the secondaries are taken from the results of Levenberg-Marquardt method applied for the IBEX-Lo ISN H map in 2009 with $\mu_{0}=0.9899$ and $\beta_{0}=$ $4.1 \times 10^{-7} \mathrm{~s}^{-1}$. The model with these boundary conditions we will call Model 4 (in addition to the three Models described in Section 5). Then, we perform fitting of the IBEX-Lo data again for all years to find the best-fitting values of $\mu_{0}$ and $\beta_{0}$. The results are presented in Fig. 6.

It is seen that 'new' values of $\mu_{0}$ and $\beta_{0}$ obtained using Model 4 are quite close to those obtained before in Section 5 using Model 1, and magnitudes of 'new' $\hat{\chi}^{2}$ for 2009-2010 are about by a factor of 2 less than 'old' $\hat{\chi}^{2}$. However, for 2011-2012 and 2017-2018 $\hat{\chi}^{2}$ is about the same as it was obtained before. Probably, the reason is the 'noise' in the data that do not allow to fit the data better. Thus, we conclude that the found parameters of the secondary interstellar atoms provide much better fit of the data in 2009-2010 (when ISN H fluxes are high) compared to the results of Model 1, while in 20112012 and 2017-2018 there are no considerable differences between the results of Model 1 and Model 4. $\mu_{0}$ and $\beta_{0}$ with corresponding uncertainties obtained by the fitting with Model 4 are summarized in Table 3. 

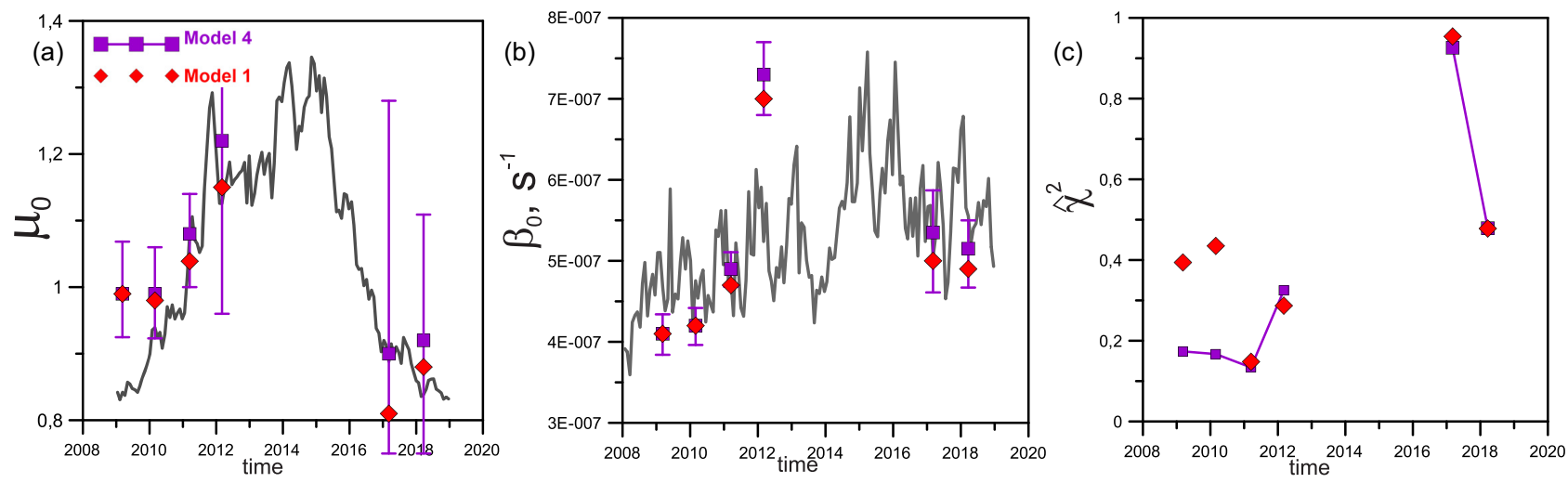

Figure 6. The same as Fig. 5, but the results of the fitting procedure are presented for Models 1 and 4. Results of the Model 1 are the same as at previous Figure and they are presented just for comparison. Uncertainties are presented only for the results of Model 4.

Table 3. Magnitudes of $\mu_{0}$ and $\beta_{0}$ derived from the fitting of IBEX-Lo data using Model 4.

\begin{tabular}{lcc}
\hline & $\mu_{0}$ & $\beta_{0} \times 10^{7} \mathrm{~s}^{-1}$ \\
\hline 2009 & $0.9899_{-0.0651}^{+0.0785}$ & $4.1_{-0.24}^{+0.26}$ \\
2010 & $0.99_{-0.067}^{+0.07}$ & $4.2_{-0.24}^{+0.24}$ \\
2011 & $1.08_{-0.08}^{+0.06}$ & $4.9_{-0.207}^{+0.22}$ \\
2012 & $1.22_{-0.26}^{+0.13}$ & $7.3_{-0.4}^{+0.5}$ \\
2017 & $0.90_{-0.15}^{+0.38}$ & $5.35_{-0.52}^{+0.74}$ \\
2018 & $0.92_{-0.17}^{+0.189}$ & $5.15_{-0.35}^{+0.48}$ \\
\hline
\end{tabular}

\section{TEMPORAL VARIATIONS OF THE ISN H FLUXES}

In this section, we consider temporal variations of the ISN $\mathrm{H}$ fluxes measured in one chosen direction with ecliptic longitude $261^{\circ}$ and ecliptic latitude $3^{\circ}$. This direction is chosen as an example because in 2009 it corresponds to maximum of the measured fluxes. For this certain direction, we extract the data points for 2013-2015 as well in addition to 2009-2012 and 2017-2018. The calculations are performed in the frame of the following models:

(i) Initial 3D time-dependent model with the ISN H parameters at 70 au taken from results of the global model IA2020 and default $\mu(t$, $\left.\lambda, w_{r}\right), \beta_{E}(t, \lambda)$ based on observational data;

(ii) $3 \mathrm{D}$ quasi-stationary version of Model 1 with $\mu_{0}$ and $\beta_{0}$ obtained by the fitting of IBEX-Lo maps in Section 5;

(iii) $3 \mathrm{D}$ quasi-stationary version of Model 4 with $\mu_{0}$ and $\beta_{0}$ obtained by the fitting of IBEX-Lo maps in Section 6.1.

The results are presented in Fig. 7. Here, we consider the fluxes measured in energy bins 1 and 2 separately and together. Note that calculations of the fluxes in the frame of Models 1 and 4 are performed for 2009-2012 and 2017-2018 because best-fitting values of $\mu_{0}$ and $\beta_{0}$ are obtained only for these years. It is seen that in 2009-2011 and 2017-2018 all models underestimate the ISN fluxes in energy bin 1 (Plot a) and overestimate the fluxes in energy bin 2 (Plot b). Model 4 provides the best agreement with the data within the error bars for the fluxes measured in energy bins 1 and 2 together (Plot c).

It is interesting to explore the ratio of the fluxes measured in bin 1 to the fluxes measured in bin 2 (Plot d). The data show increase of the ratio in 2009-2011, then sharp decrease in 2012-2015, increase again in 2017 and small decrease in 2018. The initial time-dependent
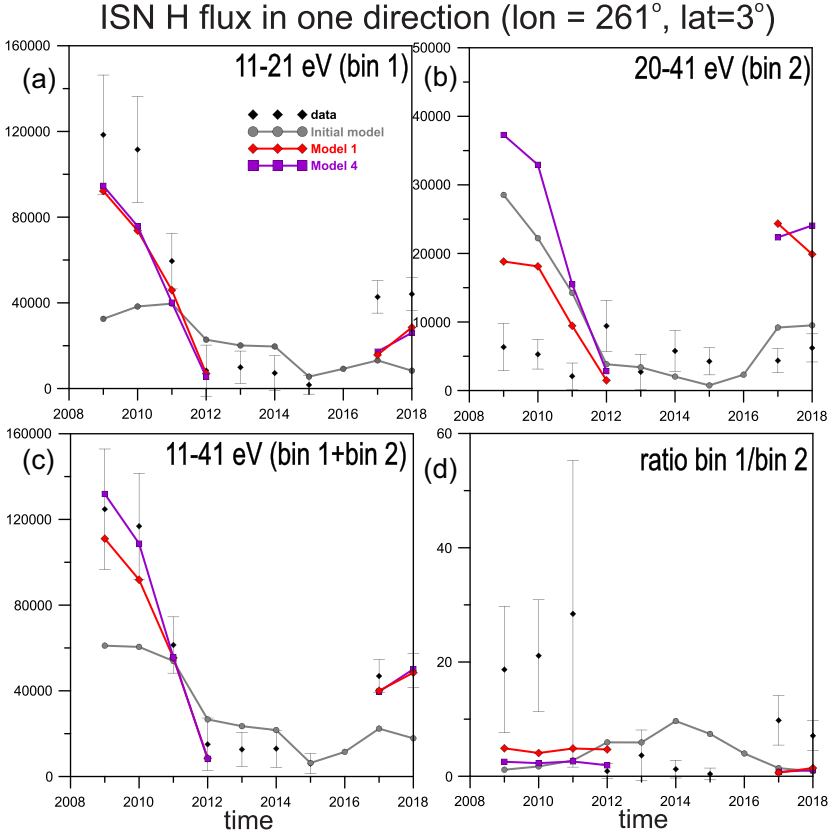

Figure 7. Temporal variations of the ISN H fluxes measured in one certain direction (ecliptic longitude $261^{\circ}$ and ecliptic latitude $3^{\circ}$ ) at energy bins 1 and 2 separately (Plots a and b), together (Plot c) and the ratio of fluxes in bins 1 to 2 (Plot d). The IBEX-Lo data are shown by the black dots. Different lines show the results of three versions of the numerical model (see text for details).

model provides smooth increase of the ratio from 2009 to 2014 and then decrease from 2014 to 2018. In 2009-2011 and 2017-2018, the model ratio is much less than in the data, while in 2012-2015 the model ratio is larger than in the data. The model behaviour of the ratio correlates with the increase and decrease of $\mu_{0}$ with time as it is seen from Fig. 6(a): $\mu_{0}$ increases from 2009 to 2014 and then decreases. Increasing of the radiation pressure means that $\mathrm{H}$ atoms become to be slower therefore hydrogen fluxes in energy bin 1 should increase and fluxes in energy bin 2 should decrease due to atoms' deceleration, so their ratio increases as it is seen in the initial model results. However, in the data we see the opposite situation. The second factor, which may influence the ratio of fluxes in bins 1 and 2 is the ionization rate. Slow atoms are more ionized than the faster ones that lead to effective increase of averaged atom's velocity (so-called kinetic 'selection' effect). Therefore, increase of the ionization rate may lead to relative 
increase of fluxes in energy bin 2 compared to fluxes in energy bin 1 and corresponding decrease of the ratio. Thus, increase of $\mu_{0}$ and $\beta_{0}$ has opposite effect on the ratio of fluxes. As we see from known ionization rates models, the averaged level of the ionization rate is about constant from 2009 to 2013 and increases in 2014 (Fig. 6b). In the IBEX-Lo data, the ratio is very small in 2012-2015. This means that the ionization rate in these years should be large. Indeed, the best-fitting value of $\beta_{0}$ found by fitting of the IBEX-Lo data in 2012 is about $7 \times 10^{-7} \mathrm{~s}^{-1}$ that is much higher than the known one. Thus, in order to explain the ratio of $\mathrm{H}$ fluxes in energy bin 1 to energy bin 2 shown in the IBEX-Lo data we need the ionization rate at 1 au to be much larger in 2012-2015 than it was thought before. Another possible explanation may be that the distribution of ISN fluxes into the two IBEX-Lo energy bins differs from ground-based calibrations. If there are problems with the determination of the atoms' energy, total fluxes measured in two energy bins together may be correct while the distribution into individual energy bins may be incorrect. We cannot exclude such a possibility. Because the current models cannot explain the ratio of the ISN H fluxes measured in energy bins 1 and 2 observed by IBEX-Lo, we argue that only the sum of the fluxes measured in the two bins together should be considered for a robust model fit.

\section{SUMMARY}

In this paper, we analysed the ISN H fluxes measured by IBEX-Lo in 2009-2012 and 2017-2018 years. We investigated the impact of $\mu$ and $\beta$ for the maps of the fluxes obtained with IBEX's observational geometry. It was found that parameter $\mu$ influence both the position of maximum fluxes and absolute values of fluxes, while parameter $\beta$ significantly influence only the absolute values of fluxes. We performed a fitting of the data by the quasi-stationary model results and found the best-fitting magnitudes of $\mu_{0}$ and $\beta_{0}$ by minimization of $\chi^{2}$ for 2009-2012 and 2017-2018. Results of the fitting procedure are about the same for three different types of the numerical models (with and without latitudinal variations of the ionization rate and with different types of the boundary conditions for the $\mathrm{H}$ velocity distribution function at 70 au from the Sun). Generally, the obtained values of $\mu_{0}$ and $\beta_{0}$ agreed within the uncertainties with those known before, except two noticeable differences.

The first difference is the magnitude of $\mu_{0}$ in 2009: the fitting procedure provides $\mu_{0}=0.98$ that is 16 per cent larger than derived from the model of Kowalska-Leszczynska et al. (2020). The similar result was obtained by Rahmanifard et al. (2019) from analysis of longitude of maximum fluxes measured by IBEX-Lo. We checked if plausible $\mathrm{H}$ parameters at $70 \mathrm{au}$ exist, which would allow for a good agreement with the IBEX-Lo data and low $\mu_{0}=0.84$ in 2009. Using Levenberg-Marquardt method we found parameters (number densities, velocity, and temperatures) of the secondary interstellar atoms at $70 \mathrm{au}$, which provide the best agreement with the IBEX-Lo data in 2009. For the model with $\mu_{0}=0.84$ the found parameters are unrealistic. Therefore, we conclude that to explain IBEX-Lo data in 2009 we certainly need larger magnitude of $\mu_{0}$. There are no options to fit the data with small value of $\mu_{0}$ using our numerical models of the ISN H distribution. Oppositely, for the model with $\mu_{0}=0.98$ the found ISN H parameters at 70 au are similar to those obtained in the upwind direction by IA2020 model. There are some differences in the averaged velocity of the secondary atoms, but they lie within quite large error bars associated with uncertainties of IBEX-Lo data.

Note that in 2009-2012 the magnitudes of $\mu_{0}$ obtained here agree very well with the results of Rahmanifard et al. (2019). For 20172018, Rahmanifard et al. (2019) have obtained a little bit large values of $\mu_{0}$, but they are within the uncertainties of our results. Let us remind that Rahmanifard et al. have used for the analysis only the position of maximum fluxes at the IBEX-Lo sky map, while we use the whole set of the fluxes themselves. The found differences in 2017-2018 may be caused by large uncertainties in the data and difficulties to determine the main maximum of fluxes on the maps as it is seen in our Fig. 2(e,f).

The second difference between the set of $\left(\mu_{0}, \beta_{0}\right)$ found here and the previously preferred set is the magnitude of $\beta_{0}$ in 2012 that is about 20 per cent larger than it was obtained before from the ionization rate models. For all other years the found $\beta_{0}$ are a bit smaller that the ones derived by previous models, but differences are within the error bars. The IBEX-Lo ISN H fluxes measured in energy bin 1 sharp decrease in 2012, and that is why the ionization rate obtained by fitting of the data is large. Decrease of the fluxes in 2012 shown in IBEX data is much stronger than in the model results. During the summer 2012, the post-acceleration voltage (PAC) of IBEX-Lo was dropped off and the calibration factor was changed accordingly (Galli et al., 2019). Most part of the ISN H measurements in 2012 are performed during the winter and spring, i.e. before the noted reduce of PAC. However, we cannot exclude some possible technical issues related with difficulties to measure very low fluxes of low energetic atoms during this period.

Analysis of the temporal variations of the ISN H fluxes measured in 2009-2018 for one fixed direction shows that the ratio of fluxes measured in energy bins 1 and 2 in the data is significantly different from what our model predicts. Namely, around solar minimum in 2009-2011 and 2017-2018 the model predicts less fluxes in energy bin 1 and more fluxes in energy bin 2 compared to the data, while around solar maximum 2012-2015 the situation is opposite. Our bestfitting Model 4 that gives the smallest values of $\chi^{2}$ shows a good agreement with the data for sum of the fluxes measured in energy bins 1 and 2 together, but cannot explain the ratio of fluxes in bins 1 to 2 observed by IBEX-Lo. The IBEX-Lo ISN H fluxes measured in energy bin 2 at the chosen direction are about the same for all years with averaged level $5300 \mathrm{~cm}^{-2} \mathrm{~s}^{-1} \mathrm{sr}^{-1}$, while in the model results the fluxes in energy bin 2 varies during the solar cycle by a factor of 500. We cannot explain these differences from physical point of view. However, uncertainties of the IBEX-Lo data at low-energy bins are quite large (here we consider the data with uncertainties up to 90 per cent). More detailed and accurate measurements are needed to explore the energy distribution of low energetic ISN $\mathrm{H}$ atoms in IBEX-Lo data. At this stage, we think that for the model analysis it is better to use sum of the fluxes measured in the two lowest energy bins rather than fluxes measured at bins 1 and 2 separately. We hope that the upcoming Interstellar Mapping and Acceleration Probe mission will provide opportunities to more accurately observe the ISN atoms near the Earth and thus to improve our understanding of the interstellar atoms in the heliosphere.

\section{ACKNOWLEDGEMENTS}

We thank all of the outstanding men and women who have made the IBEX mission such a wonderful success. Numerical calculations of the ISN H fluxes and data analysis performed by OK are supported by Russian Foundation of Basic Research (RFBR), grant no. 1832-20085. Global model of the heliosphere is obtained by DA and VI in the frame of the Russian Science Foundation grant no. 1912-00383. AG thanks the Swiss National Science Foundation for financial support. 


\section{DATA AVAILABILITY}

The IBEX-Lo data underlying this article will be shared on reasonable request to the André Galli (andre.galli@space.unibe.ch).

\section{REFERENCES}

Baliukin I. I., Izmodenov V. V., Möbius E., Alexashov D. B., Katushkina O. A., Kucharek H., 2017, ApJ, 850, 119

Baranov V. B., Malama Y. G., 1993, J. Geophys. Res., 98, 15157

Bertaux J. L., Blamont J. E., 1971, A\&A, 11, 200

Bertaux J. L., Lallement R., Kurt V. G., Mironova E. N., 1985, A\&A, 150, 1

Bzowski M., Möbius E., Tarnopolski S., Izmodenov V., Gloeckler G., 2008, A\&A, 491, 7

Bzowski M. et al., 2012, ApJS, 198, 12

Bzowski M., Kubiak M. A., Czechowski A., Grygorczuk J., 2017, ApJ, 845, 15

Costa J., Lallement R., Quémerais E., Bertaux J.-L., Kyrölä E., Schmidt W., 1999, A\&A, 349, 660

Fayock B., Zank G. P., Heerikhuisen J., Gilbert C. R., Scherer K., 2015, J. Phys.: Conf. Ser., 642, 012007

Funsten H. O. et al., 2009, Space Sci. Rev., 146, 75

Fuselier S. A. et al., 2009, Space Sci. Rev., 146, 117

Galli A. et al., 2019, ApJ, 871, 52

Geiss J., Gloeckler G., Fisk L. A., 2006, in Izmodenov V. V., Kallenbach R., eds, The Physics of the Heliospheric Boundaries ISSI Scientific Reports Series, ESA Publications Division, The Netherlands, p. 137

Gloeckler G. et al., 1993, Science, 261, 70

Izmodenov V., 2001, Space Sci. Rev., 97, 385

Izmodenov V. V., Alexashov D. B., 2015, ApJS, 220, 32

Izmodenov V. V., Alexashov D. B., 2020, A\&A, 633, L12

Izmodenov V. V., Katushkina O. A., Quémerais E., Bzowski M., 2013, ISSI Scientific Report Series, Vol. 31, Springer Science+Business Media, New York, p.7

Katushkina O. A., Izmodenov V. V., Wood B. E., McMullin D. R., 2014, ApJ, 789,80

Katushkina O. A., Izmodenov V. V., Alexashov D. B., Schwadron N. A., McComas D. J., 2015, ApJS, 220, 33

Koutroumpa D., Quémerais E., Ferron S., Schmidt W., 2019, Geophys. Res. Lett., 46, 4114

Kowalska-Leszczynska I., Bzowski M., Sokół J. M., Kubiak M. A., 2018, ApJ, 852, 115

Kowalska-Leszczynska I., Bzowski M., Kubiak M. A., Sokół J. M., 2020, ApJS, 247, 62

Kubiak M. A. et al., 2014, ApJS, 213, 29

Kubiak M. A. et al., 2016, ApJS, 223, 25

Kubiak M. A., Bzowski M., Sokół J. M., 2019, ApJ, 882, 114

Lallement R., Quémerais E., Bertaux J. L., Ferron S., Koutroumpa D., Pellinen R., 2005, Science, 307, 1447

Lallement R., Quémerais E., Koutroumpa D., Bertaux J.-L., Ferron S., Schmidt W., Lamy P., 2010, AIP Conf. Proc. Vol. 1216, Twelfth International Solar Wind Conference. Am. Inst. Phys., New York, p. 555

Machol J., Snow M., Woodraska D., Woods T., Viereck R., Coddington O., 2019, Earth Space Sci., 6, 2263

McComas D. J. et al., 2009, Science, 326, 959

McComas D. J. et al., 2015, ApJ, 801, 28

Möbius E. et al., 2004, A\&A, 426, 897

Möbius E. et al., 2009, Science, 326, 969

Möbius E. et al., 2012, ApJS, 198, 11

Opher M., Loeb A., Drake J., Toth G., 2020, Nat. Astron., 4, 675

Park J., Kucharek H., Möbius E., Galli A., Livadiotis G., Fuselier S. A., McComas D. J., 2015, ApJS, 220, 34

Park J., Kucharek H., Möbius E., Galli A., Kubiak M. A., Bzowski M., McComas D. J., 2016, ApJ, 833, 130

Park J. et al., 2019, ApJ, 880, 4

Pogorelov N. V., Borovikov S. N., Zank G. P., Ogino T., 2009, ApJ, 696, 1478
Pogorelov N. V., Heerikhuisen J., Roytershteyn V., Burlaga L. F., Gurnett D. A., Kurth W. S., 2017, ApJ, 845, 9

Press W. H., Flannery B. P., Teukolsky S. A., , Numerical recipes. The art of scientific computing, Cambridge: University Press

Pryor W. et al., 2008, A\&A, 491, 21

Quémerais E., 2000, A\&A, 358, 353

Quémerais E., Lallement R., Ferron S., Koutroumpa D., Bertaux J.-L., Kyrölä E., Schmidt W., 2006, J. Geophys. Res., 111, A09114

Rahmanifard F. et al., 2019, ApJ, 887, 217

Saul L. et al., 2012, ApJS, 198, 14

Schwadron N. A. et al., 2013, ApJ, 775, 86

Schwadron N. A. et al., 2016, ApJ, 828, 81

Sokół J. M., Bzowski M., Tokumaru M., 2019, ApJ, 872, 57

Swaczyna P., McComas D. J., Schwadron N. A., 2019, ApJ, 871, 254

Thomas G. E., Krassa R. F., 1971, A\&A, 11, 218

Witte M., 2004, A\&A, 426, 835

Zank G. P., Heerikhuisen J., Wood B. E., Pogorelov N. V., Zirnstein E., McComas D. J., 2013, ApJ, 763, 20

\section{APPENDIX A: CALCULATIONS OF THE ISN H FLUXES MEASURED BY IBEX-LO}

To compare the model results with the IBEX-Lo data, one needs to calculate the ISN $\mathrm{H}$ fluxes exactly in the same way as they are collected by IBEX. We use the real position and velocity of IBEX with resolution of $1 \mathrm{~d}$. For each orbit, we know direction of the IBEX's spin axis and find the observational plane that is perpendicular to the spin axis. Note that during each orbit the spin axis remains the same and spin angle is counting in the observational plane from the projection of the north ecliptic pole to this plane. The model fluxes are calculated daily for spin angles with resolution of $1^{\circ}$. Then, the fluxes are averaged over $6^{\circ}$ angular bins and over duration of the orbit. To calculate the model flux for certain direction, one needs to take into account integration over acceptance angles within the geometrical collimator and over corresponding energies within the certain energy bin. Thus, the ISN H flux for the energy bin $i$ and direction of the line of sight corresponding to the spin angle $\alpha_{j}$ is calculated as follows:

$$
\begin{aligned}
\text { Flux }_{i, j}= & \frac{1}{\Delta t} \int_{t_{0}}^{t_{1}} \mathrm{~d} t \frac{1}{\Delta \alpha} \int_{\alpha_{j}-\Delta \alpha / 2}^{\alpha_{j}+\Delta \alpha / 2} \mathrm{~d} \alpha \times \\
& \times \int_{0}^{\theta_{\max }} \int_{0}^{2 \pi} \hat{P}(\theta, \phi) \sin \theta \mathrm{d} \theta \mathrm{d} \phi \times \\
& \times \int_{V_{i, 1}}^{V_{i, 2}} f_{H}\left(\mathbf{r}_{0}, \mathbf{w}_{H}, t\right)\left|w_{\text {rel }}\right|^{3} E_{\text {rel }} \hat{T}_{i}\left(E_{\text {rel }}\right) \mathrm{d} w_{\text {rel }} .
\end{aligned}
$$

Here, $t_{0}$ is the time of the beginning of the orbit, $t_{1}$ is the time of the end of the orbit, $\Delta t=t_{1}-t_{0}$ is the duration of the orbit. The spin angle $\alpha$ varies within the range of $\left[\alpha_{j}-\Delta \alpha / 2, \alpha_{j}+\Delta \alpha / 2\right]$ centred at $\alpha_{j}$ with angular bin-width $\Delta \alpha=6^{\circ}$. Integration over the collimator is represented by a collimator point spread function $\hat{P}(\theta, \phi)$ that determines the probability of atom's detection inside the collimator. In our calculations, we use a simplified conical shape of the collimator (instead of a realistic hexagonal shape) as we did before in Katushkina et al. (2015). In this case, $P$ depends only on one angle $\theta$ counted from the axis of the collimator. Plot of function

Table A1. Central energies $\left(E_{\mathrm{c}}\right)$, energy ranges $\left(E_{\min }\right.$ and $\left.E_{\max }\right)$ for energy bin 1 and energy bin 2 of the IBEX-Lo sensor.

\begin{tabular}{lccc}
\hline Energy bin & $E_{\mathrm{c}}, \mathrm{eV}$ & $E_{\min }, \mathrm{eV}$ & $E_{\max }, \mathrm{eV}$ \\
\hline 1 & 15 & 11 & 21 \\
2 & 29 & 20 & 41 \\
\hline
\end{tabular}


$P(\theta)$ is presented in fig. 9 by Katushkina et al. (2015). The cone solution is $\theta_{\max }=7.8^{\circ} . \hat{P}$ is a function normalized by the following:

$$
\hat{P}(\theta)=\frac{P(\theta)}{\int_{0}^{\theta_{\max }} \int_{0}^{2 \pi} P(\theta) \sin \theta \mathrm{d} \theta \mathrm{d} \phi} .
$$

It is convenient to introduce new coordinates within the collimator: $\xi=\theta \cos \phi$ and $\psi=\theta \sin \phi$. Jacobian of this transformation is $1 / \theta$. Therefore, integration over the collimator angles $\theta$ and $\phi$ can be transformed to the integration over $\xi$ and $\psi$ by the following way:

$$
\int_{0}^{\theta_{\max }} \int_{0}^{2 \pi} \hat{P}(\theta) \sin \theta \mathrm{d} \theta \mathrm{d} \phi=\int_{-\theta_{\max }}^{\theta_{\max }} \int_{-\theta_{\max }}^{\theta_{\max }} \frac{\sin \theta}{\theta} \hat{P}(\theta) \mathrm{d} \xi \mathrm{d} \psi,
$$

where $\theta=\sqrt{\xi^{2}+\psi^{2}}$

In equation (A1), $f_{H}$ is the velocity distribution function of the ISH atoms at the point of observation $\mathbf{r}_{0}, w_{\text {rel }}$ is the atom velocity relative to the spacecraft, $\boldsymbol{w}_{H}$ is the absolute atom's velocity vector (i.e. $\boldsymbol{w}_{H}=$ $\boldsymbol{w}_{\text {rel }}+\boldsymbol{V}_{\mathrm{SC}}$, where $\boldsymbol{V}_{\mathrm{SC}}$ is the spacecraft velocity and the direction of $\boldsymbol{w}_{\text {rel }}$ is determined by the local line of sight inside the collimator); $E_{\text {rel }}=m_{\mathrm{H}} w_{\text {rel }}^{2} / 2, V_{i, 1}$ and $V_{i, 2}$ determine the boundaries of energy bin i: $E_{\min , i}=m_{\mathrm{H}} V_{i, 1}^{2} / 2$ and $E_{\max , i}=m_{\mathrm{H}} V_{i, 2}^{2} / 2, m_{\mathrm{H}}$ is the mass of an $\mathrm{H}$ atom; the boundaries of the energy ranges for bins 1 and 2 are taken from Schwadron et al. (2013) and listed in Table A1. Integration over the energy bin is performed with the normalized energy transmission function $\hat{T}_{i}(E)$ taken from Schwadron et al. (2013):

$$
\begin{aligned}
T_{i}(E) & =\exp \left(-4 \ln 2 \frac{\left(E / E_{c, i}-1\right)^{2}}{\Delta_{1}^{2}}\right) \quad \text { for } E \leq E_{c, i} \\
& =\exp \left(-4 \ln 2 \frac{\left(E_{c, i} / E-1\right)^{2}}{\Delta_{2}^{2}}\right) \text { for } E>E_{c, i},
\end{aligned}
$$

where $E_{c, i}$ is the central energy of a given energy bin (see Table A1), and $\Delta_{1}=2\left(1-E_{\min , i} / E_{c, i}\right), \Delta_{2}=2\left(1-E_{c, i} / E_{\max , i}\right)$. Function $\hat{T}$ in formula (A1) is normalized by the following:

$$
\hat{T}_{i}(E)=\frac{T_{i}(E)}{\int_{E_{\min , i}, i}^{E_{\max }} T_{i}(E) \mathrm{d} E},
$$

where integration is performed within the corresponding energy range.

As it is stated before, in this paper we consider the ISN H fluxes obtained in the energy bins 1 and 2 together. Therefore, in the model results we obtain a sum of the fluxes calculated for two energy bins.
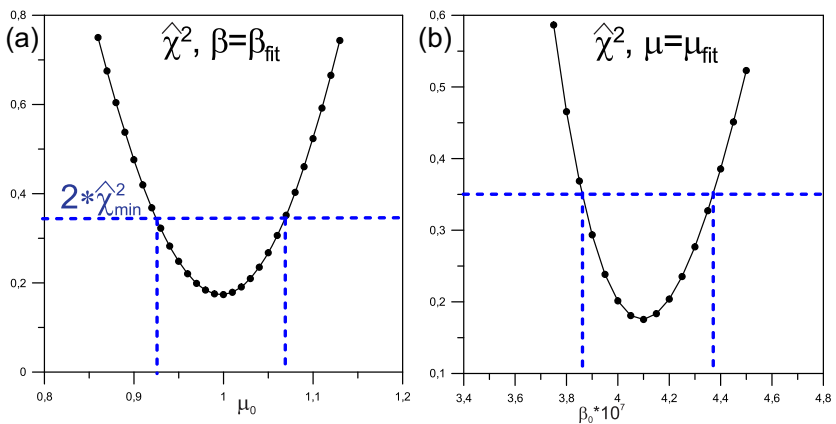

Figure B1. Example of $\hat{\chi}^{2}$ obtained by the fitting procedure of the IBEX-Lo data in 2009 using Model 4. The plots are presented 1D slices with fixed magnitude of $\beta_{0}(\mathrm{~A})$ and $\mu_{0}(\mathrm{~B})$. The dashed horizontal line shows a level of $2 \cdot \hat{\chi}_{\min }^{2}$ and the vertical dashed lines show corresponding ranges of the parameters, which provide less $\hat{\chi}^{2}$.

\section{APPENDIX B: CALCULATIONS OF UNCERTAINTIES FOR BEST-FITTING $\mu_{0}$ AND $\beta_{0}$}

Fig. B1 shows an example of obtained reduced $\chi^{2}$ as a function of $\mu_{0}$ and $\beta_{0}$. This is the result of the fitting procedure applied to the IBEX-Lo data in 2009 using Model 4. For each plot, one parameter is fixed and corresponds to the determined best-fitting magnitude and the second parameter is varied. Standard receipt for calculations of uncertainties of the found best-fitting values in the least-squares method is consideration of those parameters, which provide $\hat{\chi}^{2} \leq \hat{\chi}_{\min }^{2}+1$. However, this is true only for the case when $\hat{\chi}^{2}$ is close to 1 . For 2009 our minimum of $\hat{\chi}^{2}$ is 0.175 . Therefore, for the uncertainties we decided to choose a level of $2 \cdot \hat{\chi}_{\min }^{2}$. The dashed vertical lines at Fig. B1 show corresponding ranges of $\mu_{0}$ and $\beta_{0}$, which provide $\hat{\chi}^{2} \leq 2 \cdot \hat{\chi}_{\text {min }}^{2}$. We use this criteria for all years and find corresponding uncertainties presented in Figs 5 and 6.

This paper has been typeset from a $\mathrm{T}_{\mathrm{E}} \mathrm{X} / \mathrm{LT} \mathrm{E} \mathrm{X}$ file prepared by the author. 